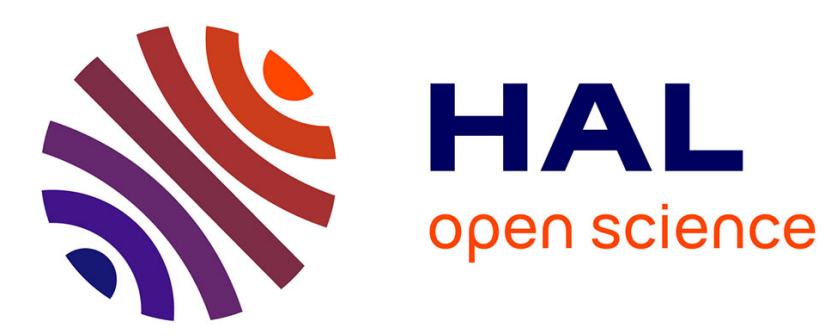

\title{
Understanding and Preventing Tacit Collusion among Telecommunication Operators
}

\author{
Patrick Maillé, Maurizio Naldi, Bruno Tuffin
}

\section{To cite this version:}

Patrick Maillé, Maurizio Naldi, Bruno Tuffin. Understanding and Preventing Tacit Collusion among Telecommunication Operators. NET-COOP, Nov 2009, Eindhoven, Netherlands. hal-00447030

\author{
HAL Id: hal-00447030 \\ https://hal.science/hal-00447030
}

Submitted on 14 Jan 2010

HAL is a multi-disciplinary open access archive for the deposit and dissemination of scientific research documents, whether they are published or not. The documents may come from teaching and research institutions in France or abroad, or from public or private research centers.
L'archive ouverte pluridisciplinaire HAL, est destinée au dépôt et à la diffusion de documents scientifiques de niveau recherche, publiés ou non, émanant des établissements d'enseignement et de recherche français ou étrangers, des laboratoires publics ou privés. 


\title{
Understanding and Preventing Tacit Collusion among Telecommunication Operators
}

\author{
Patrick Maillé ${ }^{1}$, Maurizio Naldi ${ }^{2}$, and Bruno Tuffin ${ }^{3}$ \\ 1 Institut Telecom, Telecom Bretagne \\ 2 rue de la Châtaigneraie CS 17607 \\ 35576 Cesson Sévigné Cedex, France \\ patrick.maille@telecom-bretagne.eu \\ ${ }^{2}$ Università di Roma Tor Vergata \\ Dip. di Informatica Sistemi Produzione \\ Via del Politecnico 1 \\ 00133 Roma, Italy \\ naldi@disp. uniroma2.it \\ 3 INRIA Rennes - Bretagne Atlantique \\ Campus universitaire de Beaulieu \\ 35042 Rennes Cedex, France \\ btuffin@irisa.fr
}

\begin{abstract}
Modeling the competition among telecommunication operators (or providers) as a repeated game may explain why tacit collusion to maintain high charging prices can be observed. Such outcomes clearly do not benefit the users, nor do they provide operators with incentives to improve the network quality of service to outperform their competitors. We propose a simple regulation based on price stability over time, to modify the game played by operators in a way that could prevent collusion.
\end{abstract}

Keywords: Competition, Game theory, Repeated games.

\section{Introduction}

Competition in the telecom sector has been hard since liberalization, being characterized by a number of subsequent market twists (booms and downturns), resulting e.g. in a flurry of new operators followed by market concentration periods [1. Due to the asymmetry existing between the incumbent operator and the new entrants, these have been forced to move boldly to gain market shares. Though competitors could use several leverages, telecom providers have typically acted on prices, sometimes engaging in a price war, where a price reduction by a provider is immediately followed by similar price reductions by its competitors, spurring a downward spiral [2]. Price wars are generally considered to be quite harmful for providers in the long term, since they cut the revenues of all the competitors despite the demand increase 3 . Instead, customers largely benefit from the general reduction of prices, as long as the benefit of expense reduction is not 
accompanied by worsening quality of service [4. However, Chamberlin showed that, in a scenario with a limited number of competitors, oligopolistic coordination is likely to occur, resulting in the joint maximization of profits rather than a price war 56. Cases of collusion (explicit or tacit) have been reported in the literature, both in the long distance market [7] and in the mobile sector [8] [9].

In this paper we analyse the game resulting from the competition between providers and the strategies leading to price war or collusion. Though the analysis of competition is generally dealt with in a static setting, a customer is continually confronted with the task of choosing the best provider, even on a call by call basis. Hence we consider here the competition between providers as a repeated game, where providers may continually update their prices to regain lost customers. The time aspect makes competition on prices different from a one-shot game. We show that a solution strategy in the repeated game leads to tacit collusion, hence to the prices resulting from the joint maximization of profits. We then propose imposing price stability as a regulation tool to prevent joint profit maximization from being a Nash equilibrium of the game and we also show that it can be effective to deter providers from tacit collusion.

The paper is organized as follows. Section 2 presents the demand model and the competition game that is repeatedly played between two providers, highlighting two possible outcomes of that game (the noncooperative and the cooperative ones). In Section 3, we recall the main results on repeated game theory and show how a repeated game model is well-suited to our problem, thereby leading to collusion among providers as an equilibrium. Section 4 proposes a regulatory measure based on price stability, and Section 5 investigates its effect when both providers are identical (symmetric game). Some asymmetric examples are studied in Section 6 to illustrate some particularities of the game and the proposed regulation. Conclusions and directions for future work are given in Section 7

\section{Demand Model and Competition Game among Providers}

In this paper we focus on the case of two providers. We introduce in this section the dependence of provider demands with the prices they set, and present the game played between the competing providers seeking to maximize their profit.

Assumption 1. Following [10], we consider the following demand model where demand at each provider depends on all providers' prices. Demand $D_{i}$ at provider $i \in\{1,2\}$ is given by

$$
\begin{aligned}
& D_{1}=D_{0,1}-b_{1} p_{1}+\beta_{1} p_{2} \\
& D_{2}=D_{0,2}-b_{2} p_{2}+\beta_{2} p_{1}
\end{aligned}
$$

where $D_{0, i}$ is the demand level if prices were set to zero, $b_{i}>0$ represents the negative effect of provider $i$ 's price on his demand, and $\beta_{i}>0$ the positive effect of the concurrent's price. 
Remark that the expressions in (11)-(2) can give negative demands for some values of the parameters. However for the cases we will consider (namely, noncooperative and cooperative situations among providers), the demand for each provider will be nonnegative.

It is also assumed that a uniform price increase by any firm cannot produce a total demand increase, implying $b_{1} \geq \beta_{2}$ and $b_{2} \geq \beta_{1}$. Moreover, we expect the direct effect of price $b_{i}$ on demand $D_{i}$ to be strictly larger than the indirect effect of the competitor's price $\beta_{i}$. We summarize those conditions below

$$
\forall i \neq j, \quad\left\{\begin{array}{l}
b_{i}>\beta_{i} \\
b_{i} \geq \beta_{j} .
\end{array}\right.
$$

The utilities of the providers are given by their revenues, i.e. the products price $\times$ demand:

$$
\begin{aligned}
& U_{1}\left(p_{1}, p_{2}\right)=p_{1} D_{1}=p_{1}\left(D_{0,1}-b_{1} p_{1}+\beta_{1} p_{2}\right) \\
& U_{2}\left(p_{1}, p_{2}\right)=p_{2} D_{2}=p_{2}\left(D_{0,2}-b_{2} p_{2}+\beta_{2} p_{1}\right) .
\end{aligned}
$$

Investigating the best response of provider 1 , we can see that $\partial U_{1} / \partial p_{1}=D_{0,1}$ $2 b_{1} p_{1}+\beta_{1} p_{2}$, and a maximum is obtained at $p_{1}=\left(D_{0,1}+\beta_{1} p_{2}\right) /\left(2 b_{1}\right)>0$. Similarly, the best response for provider 2 in function of provider 1 's price $p_{1}$ is $p_{2}=\left(D_{0,2}+\beta_{2} p_{1}\right) /\left(2 b_{2}\right)>0$. A Nash equilibrium (if any) is therefore a point satisfying those two equations. The system has a unique solution

$$
\left(p_{1}^{N}, p_{2}^{N}\right)=\left(\frac{2 b_{2} D_{0,1}+\beta_{1} D_{0,2}}{4 b_{1} b_{2}-\beta_{1} \beta_{2}}, \frac{2 b_{1} D_{0,2}+\beta_{2} D_{0,1}}{4 b_{1} b_{2}-\beta_{1} \beta_{2}}\right) .
$$

Remark that those prices are positive since $4 b_{1} b_{2}>\beta_{1} \beta_{2}$ from (3).

The corresponding utility values at the Nash equilibrium are denoted by $U_{1}^{N}$ and $U_{2}^{N}$, and given by

$$
\begin{aligned}
& U_{1}^{N}=b_{1}\left(\frac{2 b_{2} D_{0,1}+\beta_{1} D_{0,2}}{4 b_{1} b_{2}-\beta_{1} \beta_{2}}\right)^{2} \\
& U_{2}^{N}=b_{2}\left(\frac{2 b_{1} D_{0,2}+\beta_{2} D_{0,1}}{4 b_{1} b_{2}-\beta_{1} \beta_{2}}\right)^{2} .
\end{aligned}
$$

If we are now interested in the cooperative case where both providers try to maximize the sum of their utilities. $U\left(p_{1}, p_{2}\right)=U_{1}\left(p_{1}, p_{2}\right)+U_{2}\left(p_{1}, p_{2}\right)=p_{1}\left(D_{0,1}-\right.$ $\left.b_{1} p_{1}+\beta_{1} p_{2}\right)+p_{2}\left(D_{0,2}-b_{2} p_{2}+\beta_{2} p_{1}\right)$, the maximum is obtained when $\partial U / \partial p_{1}=$ $\partial U / \partial p_{2}=0$, i.e., $D_{0,1}-2 b_{1} p_{1}+\beta_{1} p_{2}+p_{2} \beta_{2}=0$ and $p_{1} \beta_{1}+D_{0,2}-2 b_{2} p_{2}+\beta_{2} p_{1}=0$. It yields

$$
\begin{aligned}
& p_{1}^{*}=\frac{2 b_{2} D_{0,1}+\left(\beta_{1}+\beta_{2}\right) D_{0,2}}{4 b_{1} b_{2}-\left(\beta_{1}+\beta_{2}\right)^{2}} \\
& p_{2}^{*}=\frac{2 b_{1} D_{0,2}+\left(\beta_{1}+\beta_{2}\right) D_{0,1}}{4 b_{1} b_{2}-\left(\beta_{1}+\beta_{2}\right)^{2}} .
\end{aligned}
$$

We can verify that under (3), those prices are positive, as well as the corresponding revenue for each provider. 
Remark 1. Notice that competition actually leads to a price reduction with respect to the cooperative setting. The ratio indeed gives

$$
\frac{p_{1}^{N}}{p_{1}^{*}}=\frac{4 b_{1} b_{2}-\left(\beta_{1}+\beta_{2}\right)^{2}}{4 b_{1} b_{2}-\beta_{1} \beta_{2}} \frac{2 b_{2} D_{0,1}+\beta_{1} D_{0,2}}{2 b_{2} D_{0,1}+\left(\beta_{1}+\beta_{2}\right) D_{0,2}},
$$

which is in the interval $(0,1)$ since both terms in the product are. Similarly, we can also show that $p_{2}^{N}<p_{2}^{*}$, therefore from the user point of view the noncooperative situation is preferable.

\section{Competition as a Repeated Game}

One of the aims of opening the telecommunication market and allowing several providers was to induce competition that would be beneficial for the end users. Indeed, with the possibility of losing customers, providers are incentivized to improve their quality of service, and minimize their costs so as to propose attractive prices to users, in order to retain them or attract new customers. That pricing aspect is illustrated in the previous section: if the two providers play noncooperatively (i.e. under competition), then the prices set are lower than if providers are actually owned by the same entity and maximize the sum of their revenues. Here, competition intends to make providers play a so-called "prisoner's dilemma" game [11, with a unique Nash equilibrium that does not optimize the sum of their revenues: providers have interest in cooperating so as to maximize that sum [5], but then the temptation to cheat (i.e., decrease one's price to attract more customers) is very strong.

The providers being trapped in lowering prices results in benefits to users, and in that sense is a socially desirable outcome. A possible way to quantify the performance of an outcome is the total amount of demand that is served, that is the number of users that find the price of the service low enough and subscribe. In that sense, the Nash outcome of the pricing game outperforms the joint-maximization one: from (11)-(2) the sum of the demands $D_{1}+D_{2}$ is

$$
D_{1}+D_{2}=D_{0,1}+D_{0,2}-\left(b_{1}-\beta_{2}\right) p_{1}-\left(b_{2}-\beta_{1}\right) p_{2},
$$

which is nonincreasing in both prices from (3). Since we saw in Remark 1 that $p_{i}^{N}<p_{i}^{*}$ for each provider $i$, then total demand with Nash prices is larger than with joint revenue maximization prices.

However, the pricing game described in the previous section does not take into account the time aspect: the game among providers is not played only once, but over several time periods (days, months or years). In general, user demand does not immediately adapt to prices, because switching providers may take time. However, users may choose their provider only for a specific service session (say, to connect to a WiFi hotspot). In that case, it is reasonable to assume that there is no delay in user reaction to provider price changes. In this paper, we consider that framework, and therefore assume that at each time period the same game is repeated. In such a case, joint-maximization, that we interpret as collusion among providers, may indeed occur despite the incentives to behave non-cooperatively in the one-shot game. Fisher [12] summarizes that issue: 
The study of any real oligopoly has largely become the study of how the joint-optimization solution is or is not achieved and the reasons why.[...] Tacit collusion is only made possible by the fact that the game, or games like it, will be played again.

A provider strategy in the repeated game is now a function that at each time period, associates an action (or a distribution over the possible actions) to the one-shot game strategies that have been played so far 1 . That set of previously played strategies is called the history of the game. Morerover, at each time period the players could make their action choice also dependent on some public signal, i.e. they can play correlated strategies [14]. We will not explicitly use such correlated strategies, so we do not enter the details here.

Provider $i$ 's utility for the repeated game is now defined as the sum of the utilities that he obtained at each time period, where a per-period discount factor $\delta \leq 1$ is used to compare gains obtained at different times. Consequently, if we denote by $s_{t}$ the set of strategies for the one-shot game that are played at time $t$, the normalized 2 expected utility of provider $i$ is

$$
V_{i}=(1-\delta) \sum_{t=0}^{+\infty} \delta^{t} U_{i}\left(s_{t}\right)
$$

The discount factor represents the "patience" of the players: the smaller it is, the more players value present gains against future ones. That factor $\delta$ can have different interpretations:

- it can represent the price in the current period of a monetary unit in the next period: if a per-period interest rate $r>0$ is applied, then this gives $\delta=\frac{1}{1+r}$.

- Independently of interest rates, the factor $\delta$ can also stand for the probability of the game continuing at next period. Indeed, especially in telecommunication networks, the rapid evolution of technologies and services may lead to some services being abandoned by customers, consequently ending the competition game among providers.

- The most realistic interpretation is to consider that $\delta$ represents both of those aspects, and could for example be expressed as

$$
\delta=\frac{1}{1+r} \mathbb{P}(\text { game continues at next period }) .
$$

\subsection{General Results on Repeated Games}

In this subsection, we recall the main existing results on repeated games that are related to our problem. We particularly focus on different versions of the

\footnotetext{
$\overline{1}$ Remark that in general, mixed strategies are not directly observable, since only actions are observed. Nevertheless the general results given in the next section also hold when only actions are observed 13 .

${ }^{2}$ We add the multiplicative constant $(1-\delta)$, so that the sum in (7) gives the weighted mean of the player utilities over time periods, with weight $\delta^{t}$ for period $t$.
} 
so-called Folk Theorem, that investigates the possible outcomes of a repeated game. Consider a game with $N$ players, that is repeated over time periods. For the simple game, the set of actions of each player $i$ is $A_{i}$, and we represent the actions of all players as a vector $a:=\left(a_{1}, \ldots, a_{N}\right) \in \prod_{i=1}^{N} A_{i}$ where $a_{i} \in A_{i}$ is the action of player $i$.

We denote by $U_{i}^{\text {min }}$ the minimax utility of player $i$, i.e. the minimal utility that he can ensure when the other players try to minimize $i$ 's utility:

$$
U_{i}^{\min }:=\min _{a_{-i} \in A_{-i}} \max _{a_{i} \in A_{i}} U_{i}\left(a_{i}, a_{-i}\right)
$$

where $a_{-i}$ (resp. $A_{-i}$ ) stands for the actions (resp. set of possible actions) of players different from $i$, and we write the overall action vector as $a=\left(a_{i}, a_{-i}\right)$. Consequently, $U_{i}^{\min }$ is the worst utility that $i$ can ensure if he knows the actions of his opponents. It can also be interpreted as the worst utility that the other players can impose on player $i$, and the corresponding strategies that they play to do so are called the minimax strategies against player $i$.

We also denote by $\mathbf{U}$ the set of "reachable" utility vectors of the simple game, i.e.

$$
\mathbf{U}:=\left\{\left(U_{1}(a), \ldots, U_{N}(a)\right): a \in \prod_{i=1}^{N} A_{i}\right\}
$$

The basic version of the Folk Theorem is as follows.

Theorem 1 (The Folk Theorem). For any $\left(v_{1}, \ldots, v_{N}\right)$ in the convex hull of $\mathbf{U}$, such that $v_{i}>U_{i}^{\min }$, if $\delta$ is sufficiently close to 1 then there exists a Nash equilibrium of the infinitely repeated game where, for all $i, V_{i}=v_{i}$.

The proof is simple: the utility vector $\left(v_{1}, \ldots, v_{N}\right)$ is reachable via a (correlated) strategy vector $\left(s_{1}, \ldots, s_{N}\right)$ (see for example [15] for a proof). The Nash strategies consist in each player $i$ playing $s_{i}$ while everybody does, and if a player $j$ deviates then all other players should minimize his utility forever, playing the minimax strategy against $j$.

However, in repeated games the notion of Nash equilibrium might not be strong enough: for example the previously described Nash strategies impose each player to sanction the first deviator forever, although this might be costly to them. Therefore, there might be no incentive to sanction the deviators. As a result, for a potential deviator the threat of being sanctioned might not be credible, and the deviator can reasonably expect that he will not be sanctioned.

For that reason, we use the stronger equilibrium concept of perfect (or subgame perfect) Nash equilibrium. A perfect Nash equilibrium strategy should be a Nash strategy for any subgame of the game, i.e. in any situation that can be attained. Therefore, if there exists a perfect Nash equilibrium implying sanctions, then it includes incentives for the sanctioning players to actually perform the sanctions.

Below we give three versions of the Folk Theorem involving perfect equilibria, with different hypotheses. 
Theorem 2 (Aumann-Shapley [16]/Rubinstein [17]). If $\delta=1$ then the Folk Theorem holds with perfect equilibrium.

Theorem 3 (Friedman [18]). For all $v=\left(v_{1}, \ldots, v_{N}\right)$ in the convex hull of $\mathbf{U}$, if $v_{i}>U_{i}\left(a^{N}\right)$ where $a^{N}$ is a Nash equilibrium of the simple game, then if $\delta$ is sufficiently close to 1 , there exists a perfect Nash equilibrium of the infinitely repeated game where, for all $i, V_{i}=v_{i}$.

Theorem 4 (Fudenberg and Maskin [13]). With two players, the Folk Theorem holds with perfect equilibrium. Moreover, for more than three players, if the convex hull $V^{*}$ of the reachable utility vectors dominating the minimax utilities is of dimensionality $N$, then for any $\left(v_{1}, \ldots, v_{N}\right) \in V^{*}$, if $\delta$ is sufficiently close to 1 then there exists a perfect equilibrium of the infinitely repeated game in which player $i$ 's average payoff is $v_{i}$.

The proofs for those results are built the same way: the perfect equilibrium strategy consists of playing at each period the same strategy that gives utility vector $v$ until someone deviates, and following a sanction procedure against the first deviator. The constraint of the equilibrium being perfect makes the sanction procedure more complex than for the classical version of the Folk Theorem. We do not enter the details of those strategies in this paper.

\subsection{Implications for Our Problem}

If we consider that the only pure strategies available to the providers are the Nash and joint-maximization prices exhibited in Section 2, then the one-shot game is a somehow classical prisoner's dilemma game, where the minimax sanctioning strategies are actually the Nash strategies of the one-shot game. Since it is not likely that absolutely no discounting occurs, Theorem 2 does not apply. However, it is actually realistic that the per-period discount rate be very close to 1 , because interest rates are low and/or periods are short. Therefore Folk Theorem versions in Theorems 3 and 4 apply to our model.

In particular, we focus on the particular outcome of the repeated game corresponding to tacit collusion between providers, i.e. the joint-maximization prices. Both Theorems 3 and 4 imply that if the parameter $\delta$ is sufficiently close to 1 , then providers playing joint-maximization prices at each period is a subgameperfect Nash outcome of the repeated game. This is undesirable from the point of view of users, since it means competition does not play its role in terms of price reduction.

If $\delta$ is large enough, it is very likely that the joint-maximization outcome is the Nash equilibrium that occurs among the infinity of possible perfect Nash equilibria. In the rest of the paper, we therefore look for ways to prevent such tacit collusion between the providers, by using regulatory tools.

\section{Imposing Price Stability to Prevent Collusions}

We propose here to build a regulation tool that would prevent joint-maximizing prices from being a perfect Nash equilibrium of the game played between providers. 
To do so, the regulation that is applied needs to modify the game perceived by providers, so that the hypotheses of Theorems 3 and 4 do not hold anymore. Both theorem assumptions (as well as the classical Folk Theorem) involve a $\delta$ that is sufficiently close to 1 , so those theorems do not apply anymore if we manage to reduce the value of $\delta$. The value of $\delta$ is not chosen by the regulator, but is a fixed value perceived by providers, that cannot be affected by regulator's decision. However, the regulatory measure that we propose has an effect that is equivalent to reducing the value of $\delta$ when $\delta<1$.

We suggest that the regulator imposes some stability in the provider price decisions. More precisely, providers should be allowed to modify their price every $k$ periods instead of every period, where $k$ is the stability constraint over time, fixed by the regulator. This can be written formally as follows: for each provider $i$ and each $m \in \mathbb{N}$ we impose price stability for a duration of $k$ periods, i.e.

$$
a_{k m+\ell}=a_{k m} \quad \forall m \in \mathbb{N}, i=1, \ldots, N, \ell<k .
$$

Now rewrite the objective function (overall utility) of each player $i$ :

$$
\begin{aligned}
V_{i} & =(1-\delta) \sum_{t=0}^{\infty} \delta^{t} U_{i}\left(a_{t}\right) \\
& =(1-\delta) \sum_{m=0}^{\infty} \delta^{k m} \sum_{\ell=0}^{k-1} \delta^{\ell} U_{i}\left(a_{k m+\ell}\right) \\
& =(1-\delta) \sum_{\ell=0}^{k-1} \delta^{\ell} \sum_{m=0}^{\infty} \delta^{k m} U_{i}\left(a_{k m}\right) \\
& =\left(1-\delta^{k}\right) \sum_{m=0}^{\infty}\left(\delta^{k}\right)^{m} U_{i}\left(a_{k m}\right),
\end{aligned}
$$

where the third line comes from (8). We find the same expression as in (7), but with $\delta^{k}$ instead of $\delta$ as the discount factor.

As a result, the price stability measure has the same effect on providers as a change of $\delta$ into $\delta^{k}$, since the repeated game strategies only affect the actions at time periods $(\mathrm{km})_{m \in \mathbb{N}}$. Therefore, if $\delta<1$, then the provider's "new perceived value" of the discount factor can be made as close to 0 as needed, through an appropriate choice of $k$. Notice however that introducing too much rigidity will be badly perceived by provider 3 , so $k$ should be chosen just large enough to prevent tacit collusion, as we will see next.

In what follows, we will keep the same notation for $\delta$ to designate the perceived discount factor, but we now consider the value of $\delta$ as a strategic variable for the regulator (who can reduce the original value of $\delta$ to $\delta^{k}$ ).

${ }^{3}$ On the contrary, end users may prefer such price stability to fast time-varying tariffs. 


\section{Study of the Symmetric Competition Game}

We study here our regulatory proposition, in the case when the game is perfectly symmetric. Moreover, we impose a condition on the relative values of the direct and indirect price influence factors, i.e. respectively, the $b_{i}$ and $\beta_{i}$ in (1) and (2).

Assumption 2. We consider the linear demand model described in (1)-(2), and assume that both providers are identical:

$$
D_{0,1}=D_{0,2}, \quad b_{1}=b_{2}, \quad \beta_{1}=\beta_{2} .
$$

Let us denote by $D_{0}, b$ and $\beta$ those common values for $D_{0, i}, b_{i}$ and $\beta_{i}$, respectively.

Moreover, assume that the influence factor $\beta$ of one provider's price over the demand of his opponent is sufficiently low with respect to the direct influence over his own demand, more precisely

$$
\beta<\frac{2}{3} b .
$$

In that case, the game that is repeated has symmetric Nash prices

$$
p_{1}^{N}=p_{2}^{N}=\frac{D_{0}}{2 b-\beta}:=p^{N}
$$

leading to provider revenues

$$
U_{1}^{N}=U_{2}^{N}=b\left(\frac{D_{0}}{2 b-\beta}\right)^{2}:=U^{N} .
$$

On the other hand, joint-maximizing prices are

$$
p_{1}^{*}=p_{2}^{*}=\frac{D_{0}}{2 b-2 \beta}:=p^{*},
$$

and the corresponding provider revenues are

$$
U_{1}^{*}=U_{2}^{*}=\frac{D_{0}^{2}}{4(b-\beta)} .
$$

We have $p^{*}>p^{N}$ as proved before in the general case.

We now assume that providers only have the choice between playing price $p^{N}$ or $p^{*}$. In cases when one provider plays $p^{N}$ and the other $p^{*}$, the one playing the Nash price gets revenue

$$
U_{1}\left(p^{N}, p^{*}\right)=U_{2}\left(p^{*}, p^{N}\right)=D_{0}^{2} \frac{2 b^{2}+\beta^{2}-2 b \beta}{(2 b-2 \beta)(2 b-\beta)^{2}},
$$

while the opponent gets revenue

$$
U_{2}\left(p^{N}, p^{*}\right)=U_{1}\left(p^{*}, p^{N}\right)=D_{0}^{2} \frac{2 b^{2}-3 b \beta}{(2 b-\beta)(2 b-2 \beta)^{2}} .
$$




\begin{tabular}{|c|c|c|}
\cline { 2 - 3 } \multicolumn{1}{c|}{} & $p^{N}$ & $p^{*}$ \\
\hline$p^{N}$ & $(0,0)$ & $\left(\frac{\beta^{2}}{2 b(b-\beta)},-\frac{\beta^{2}}{(2 b-2 \beta)^{2}}\right)$ \\
\hline$p^{*}$ & $\left(-\frac{\beta^{2}}{(2 b-2 \beta)^{2}}, \frac{\beta^{2}}{2 b(b-\beta)}\right)$ & $\left(\frac{\beta^{2}}{4 b(b-\beta)}, \frac{\beta^{2}}{4 b(b-\beta)}\right)$ \\
\hline
\end{tabular}

Fig. 1. The one-shot competition game among symmetric providers (under Assumption 2), with relative utilities $X=\left(U-U^{N}\right) / U^{N}$. Lines and columns represent the strategic choices of provider 1 and 2, while the values in the table are of the form $\left(X_{1}, X_{2}\right)$.

We normalize the revenues at those outcomes through the linear transformation $X=\left(U-U^{N}\right) / U^{N}$. The results therefore give relative revenue values with respect to the Nash equilibrium utilities of the one-shot game. They are summarized in Figure 1.

Interestingly, the relative (with respect to the one-shot game Nash equilibrium) provider utilities depend only on the price sensitivities $b$ and $\beta$, regardless of the value of $D_{0}$. Therefore, to study the game and set an appropriate value for the price stability duration, the regulator only needs to evaluate those sensitivities, and the per-period discount factor $\delta$.

Moreover, notice that $\frac{\beta^{2}}{(2 b-2 \beta)^{2}}>\frac{\beta^{2}}{4 b(b-\beta)}$ whenever $\beta<b$, so that we can write

$$
\frac{\beta^{2}}{(2 b-2 \beta)^{2}}=(1+\alpha) \frac{\beta^{2}}{4 b(b-\beta)}
$$

with $\alpha>0$.

We simplify again the writing of game without changing the relative preference of providers, by dividing all utilities by the constant factor $\frac{\beta^{2}}{4 b(b-\beta)}$. That normalization of utilities yields the game depicted in Figure 2,

We can then apply Theorem 3 to the one-shot game depicted in Figure 2, If $\delta$ is large enough, joint-maximization can be attained by the following perfect Nash strategy for each provider.

\begin{tabular}{|c|c|c|}
\cline { 2 - 3 } \multicolumn{1}{c|}{} & $p^{N}$ & $p^{*}$ \\
\hline$p^{N}$ & $(0,0)$ & $(2,-1-\alpha)$ \\
\hline$p^{*}$ & $(-1-\alpha, 2)$ & $(1,1)$ \\
\hline
\end{tabular}

Fig. 2. Simplified writing of the one-shot competition game among symmetric providers (under Assumption 2) 
- Play $p^{*}$ while both do,

- If one provider deviates, then play $p^{N}$ forever.

This is a Nash equilibrium if the sanction in future periods for being in the situation $\left(p^{N}, p^{N}\right)$ instead of $\left(p^{*}, p^{*}\right)$ exceeds the immediate gain for deviating. The sanction in future periods equals $\left.(1-0) \times \sum_{m=1}^{\infty} \delta^{m}=\delta /(1-\delta)\right)$, while the immediate gain for a deviator is $2-1=1$, therefore if $\delta /(1-\delta)>1$, or equivalently $\delta>1 / 2$, then the strategy described before is a perfect Nash strategy.

On the contrary, if $\delta<1 / 2$ then no outcome other than $\left(p^{N}, p^{N}\right)$ can be sustained as a perfect Nash equilibrium of the repeated game. Indeed, any point $\left(U_{1}, U_{2}\right)$ in the convex hull of $\mathbf{U}$ defined in Subsection 3.1 is such that $U_{1}+U_{2} \leq 2$, therefore necessarily at least one of the two providers expects an average weighted revenue less than 1 at a perfect Nash equilibrium. Consequently for that provider, switching from $p^{N}$ to $p^{*}$ represents an immediate gain larger than 1 , and a total future sanction lower than $\delta /(1-\delta)<1$, which implies that he will always prefer playing $p^{N}$. Anticipating that, the competitor will also play $p^{N}$.

Recall that $\delta$ is actually close to 1 in real situations, but we can artificially reduce it through the price stability regulation policy explained before. For the game we are studying, if we choose a "price stability period" $k$ such that $\delta^{k}<1 / 2$ then no collusion should occur. We therefore have a threshold on the stability period $k$ to prevent collision from occurring as a (perfect) Nash equilibrium of the repeated game.

Proposition 1. Consider a repeated competition game between two identical providers. Under Assumptions 1 and 2, then the high-prices situation $\left(p^{*}, p^{*}\right)$ can be attained as a Nash equilibrium of the regulated repeated game between providers if and only if the price stability duration $k$ is such that $\delta^{k} \geq 1 / 2$.

On the contrary, if $\delta^{k}<1 / 2$, then the only Nash equilibrium of the regulated repeated game is for each provider to play $p^{N}$ at each period.

If we consider a quite stable technology, i.e. we neglect the probability that the technology be abandoned at the next time period, then the value of $\delta$ should be determined by the interest rate $r$, that we can take close to $5 \%$ per year to fix ideas. This would give a per year discount factor of $\delta=\frac{1}{1+r}=0.95$. With such a value, remark that the price stability period imposed by the regulator should be of the order of $k=15$ years so that the "regulated" discount factor $\delta^{k}$ become lower than $1 / 2$. Such a rigidity imposed by the regulator is much too strong to be acceptable, implying that for a perfectly symmetric game between providers, the regulation method we are suggesting cannot be applied to prevent collusion. However, we will see next in Subsection 6.2 that regulating through the imposition of a reasonable price stability can be efficient to elicit providers to actually play the competition game instead of colluding.

${ }^{4}$ We assume that a reasonable price stability period should be less than one year, or two at most. 


\section{Some Numerical Examples}

In this section, we point out two specific examples where the game is not symmetric, that highlight different phenomena.

\subsection{Joint-Maximization Is Not Always Beneficial to Both Providers}

Consider the situation where $\left(D_{0,1}, D_{0,2}\right)=(1,2)$, that can be interpreted as provider 2 being able to reach more customers (e.g. through a more extended coverage region). Then it can also seem realistic to consider that the price effects modeled by $b_{i}$ and $\beta_{i}, i=1,2$, are also proportional to the covered population since only it can be affected by price changes. We take here the case where $b_{i}=$ $2 \beta_{i}=\alpha D_{0, i}$ with a strictly positive constant $\alpha$. Remark that $\alpha$ has no influence over normalized utilities, since it just depends on the price unit chosen; likewise, the influence on absolute utilities (revenues) is only through a multiplicative constant and does not affect the analysis of the game.

We give in Figure 3 the absolute utility values for the one-shot game with $\alpha=1$. Interestingly, while the price profile $\left(p_{1}^{*}, p_{2}^{*}\right)$ maximizes the sum of the

\begin{tabular}{|c|c|c|}
\cline { 2 - 3 } \multicolumn{1}{c|}{} & $p_{2}^{N}$ & $p_{2}^{*}$ \\
\hline$p_{1}^{N}$ & $(0.44,0.88)$ & $(0.54,0.72)$ \\
\hline$p_{1}^{*}$ & $(0.14,1.25)$ & $(0.38,1.25)$ \\
\hline
\end{tabular}

Fig. 3. Provider utilities when $\left(D_{0,1}, D_{0,2}\right)=(3,4)$ and $b_{i}=2 \beta_{i}=D_{0, i}, i=1,2$

provider revenues, we remark that provider 1 would prefer the profile $\left(p_{1}^{N}, p_{2}^{N}\right)$ in terms of its own revenue. Therefore the collaborative price profile $\left(p_{1}^{*}, p_{2}^{*}\right)$ is not sustainable as a Nash equilibrium of the repeated game. Possibly other kinds of collusions could occur, but they would imply some cycles in price strategies (e.g. provider 1 agrees to play $p_{1}^{*}$, in the exchange for provider 2 to tolerate a period where $\left(p_{1}^{N}, p_{2}^{*}\right)$ later on), or mixed pricing strategies. Both possibilities are not realistic outcomes for a telecommunication market, where frequent price changes are badly perceived by customers. Therefore in such situations, the most likely outcome is that providers play at each time period the Nash equilibrium prices of the one-shot game, i.e. no collusion occurs.

\subsection{A Limited Regulation Period May Be Sufficient to Prevent Collusions}

Consider the same situation as in the previous subsection, but where the asymmetry between providers is less marked. We assume that $\left(D_{0,1}, D_{0,2}\right)=(3,4)$, and still consider that $b_{i}=2 \beta_{i}=\alpha D_{0, i}$ with $\alpha>0$.

The absolute (for the case $\alpha=1$ ) and normalized provider utilities for the four outcomes of the one-shot game are given in Figure 4 and 5 , respectively. 


\begin{tabular}{|c|c|c|}
\cline { 2 - 3 } \multicolumn{1}{c|}{} & $p_{2}^{N}$ & $p_{2}^{*}$ \\
\hline$p_{1}^{N}$ & $(1.33,1.78)$ & $(1.63,1.42)$ \\
\hline$p_{1}^{*}$ & $(0.86,2.30)$ & $(1.34,2.18)$ \\
\hline
\end{tabular}

Fig. 4. Provider utilities when $\left(D_{0,1}, D_{0,2}\right)=(3,4)$ and $b_{i}=2 \beta_{i}=D_{0, i}, i=1,2$

\begin{tabular}{|c|c|c|}
\cline { 2 - 3 } \multicolumn{1}{c|}{} & $p_{2}^{N}$ & $p_{2}^{*}$ \\
\hline$p_{1}^{N}$ & $(0,0)$ & $(64.45,-0.87)$ \\
\hline$p_{1}^{*}$ & $(-101.76,1.29)$ & $(1,1)$ \\
\hline
\end{tabular}

Fig. 5. Normalized utilities when $\left(D_{0,1}, D_{0,2}\right)=(3,4)$ and $b_{i}=2 \beta_{i}=\alpha D_{0, i}, i=1,2$

We remark in Figure 4 that the gain of collusion is quite low for provider 1 (less than $1 \%$ utility improvement) with respect to the noncooperative outcome $\left(p_{1}^{N}, p_{2}^{N}\right)$, while it represents a revenue increase of more than $20 \%$ for provider 2 . On the other hand, the incentive to cheat is strong for provider 1. Consequently, the collusion outcome $\left(p_{1}^{*}, p_{2}^{*}\right)$ can only be sustained if the immediate gain to provider of playing $p_{1}^{N}$ instead of $p_{1}^{*}$ is lower than the loss in the remaining time periods. From Figure 5, this means that collusion can occur only if $(64.45-1) \leq$ $\frac{\delta^{k}}{1-\delta^{k}}$, or equivalently $\delta^{k} \geq \frac{63.45}{64.45}=0.9845$ if providers are allowed to change prices every $k$ time periods. Remark that provider 2 is much more incentivized to keep on collaborating with his competitor, since the gain from collusion is large, and the temptation to cheat is quite low.

We assume that the game can be played on a monthly basis. Under that assumption, the probability that the technology be abandoned the period after is very low in general, while the interest rate can be reasonably taken of the order of $0.5 \%$ per month. Therefore, a monthly discount factor of $\delta=\frac{1}{1+0.005}=0.995$ is reasonable. From the reasoning above, such a discount factor is sufficiently high to sustain collusion. But we also remark that the regulatory method based on price stability manages to prevent collusion as soon as prices have to be fixed for 4 months.

Therefore in that situation, the regulation method we are considering in this paper can be effective against provider collusion, with a limited flexibility restriction imposed on providers.

\section{Conclusions}

We have proposed to model competition between telecommunication service providers as a repeated noncooperative game, in order to understand some tacit collusion phenomena that have been recently observed in practice. We have remarked that the time aspects allows providers to build credible threats to each 
other so as to maintain high prices, even if each provider could improve its short-term revenue by a price decrease.

A simple regulation tool imposing some price stability could make the sanction threat between providers less efficient, and could thereby in some cases prevent high price situations to occur as a Nash equilibrium of the repeated game.

However, for some other cases (like when the game is perfectly symmetric), only a prohibitively long price stability period could be efficient against collusion. Some other regulation mechanisms need thus be found to make competition benefit to users in those cases.

\section{Acknowledgments}

The authors would like to acknowledge the support of Euro-NF Network of Excellence through the specific research project PRECO, and the French research agency through the CAPTURES project.

\section{References}

1. Cheng, J.Z., Tsyu, J.Z., Yu, H.C.D.: Boom and gloom in the global telecommunications industry. Technology in Society 25(1), 65-81 (2003)

2. Bergen, M.E., Rao, A.R., Davis, S.: How to fight a price war. Harvard Business Review 78(2), 107-116 (2000)

3. Brandenburger, A.M., Nalebuff, B.J.: Co-Opetition: A Revolution Mindset That Combines Competition and Cooperation: The Game Theory Strategy That's Changing the Game of Business, 1st edn. Doubleday Business (1997)

4. Currier, K.M.: A practical approach to quality-adjusted price cap regulation. Telecommunications Policy 31(8-9), 493-501 (2007)

5. Chamberlin, E.H.: A Theory of Monopolistic Competition. Cambridge, Massachusets (1933)

6. Fellner, W.: Competition among the Few: Oligopoly and Similar Market Structures. Alfred A. Knopf, New York (1949)

7. MacAvoy, P.W.: Tacit Collusion Under Regulation in the Pricing of Interstate Long-Distance Telephone Services. Journal of Economics \& Management Strategy 4(2), 147-185 (1995)

8. Massey, P., McDowell, M.: Joint Dominance and Tacit Collusion: An Analysis of the Irish Vodafone/O2 Case and the Implications for Competition and Regulatory Policy. Working Paper WP08/05, UCD Centre for Economics Research, University College Dublin (2008)

9. Grzybowski, L., Karamti, C.: Competition in Mobile Telephony in France and Germany. Working Paper Series Working Paper 07-24, NET Institute (2007)

10. Allon, G., Federgruen, A.: Service competition with general queueing facilities. Operations Research 56(4), 827-849 (2008)

11. Fudenberg, D., Tirole, J.: Game Theory. MIT Press, Cambridge (1991)

12. Fisher, F.M.: Games economists play: A noncooperative view. The RAND Journal of Economics 20(1), 113-124 (1989)

13. Fudenberg, D., Maskin, E.: The folk theorem in repeated games with discounting or with incomplete information. Econometrica 54(3), 533-554 (1986) 
14. Aumann, R.: Subjectivity and correlation in randomized strategies. Journal of Mathematical Economics 1(1), 67-96 (1974)

15. Osborne, M.J., Rubinstein, A.: A Course in Game Theory. MIT Press, Cambridge (1994)

16. Aumann, R., Shapley, L.: Long term competition: A game theoretic analysis. Mimeo, Hebrew University (1976)

17. Rubinstein, A.: Equilibrium in supergames with the overtaking criterion. Journal of Economic Theory 21, 1-9 (1979)

18. Friedman, J.: A noncooperative equilibrium for supergames. Review of Economic Studies 38, 1-12 (1971) 